archives-ouvertes

\title{
The novel Artificial Neural Network assisted models: A review \\ Bhanu Srivastav
}

\section{To cite this version:}

Bhanu Srivastav. The novel Artificial Neural Network assisted models: A review. 2021. hal-03158440

\section{HAL Id: hal-03158440 \\ https: / hal.archives-ouvertes.fr/hal-03158440}

Preprint submitted on 3 Mar 2021

HAL is a multi-disciplinary open access archive for the deposit and dissemination of scientific research documents, whether they are published or not. The documents may come from teaching and research institutions in France or abroad, or from public or private research centers.
L'archive ouverte pluridisciplinaire HAL, est destinée au dépôt et à la diffusion de documents scientifiques de niveau recherche, publiés ou non, émanant des établissements d'enseignement et de recherche français ou étrangers, des laboratoires publics ou privés. 


\section{The novel Artificial Neural Network assisted models: A review Bhanu Srivastav}

Canara Bank

March, 2021

\section{Abstract:}

Neural networks are one of the methods of artificial intelligence. It is founded on an existing knowledge and capacity to learn by illustration of the biological nervous system. Neural networks are used to solve problems that could not be modeled with conventional techniques. A neural structure can be learned, adapted, predicted, and graded. The potential of neural network parameters is very strong prediction. The findings are more reliable than standard mathematical estimation models. Therefore, it has been used in different fields.

This research reviews the most recent advancement in utilizing the Artificial neural networks. The reviewed studies have been extracted from Web of Science maintained by Clarivate Analytics in 2021. We find that among the other applications of ANN, the applications on Covid-19 are on the rise.

Keywords: ANN, Covid-19, Dust, Gas, Organic richness

\section{Introduction}

A neural network can be described as a human brain-based reasoning model. A tightly connected collection of nerve cells or simple data processing units, or neurons, is used in the brain. The human brain has about 10 billion neurons within them and 60 trillion synapses. The brain can accomplish its purpose even quicker than the fastest computers in the world by using several neurons simultaneously. Every neuron has a very basic structure, but an army is a great computing force. [1] A neuron consists of a cell, a soma, multiple fibers known as dendrites and a small, long fiber known as the axon.

Although Dendrites branch along the soma, the axon spreads to other neurons' dendrites and somas. The schemas of a neural network is seen in Figure 1. Complex electrochemical reactions propagate signals from one neuron to another. The electrical potencies of the cell body are 
modified by chemical compounds emitted from the synapses. [2] As the potential exceeds its threshold, the axon produces an electric pulse, an action force. The pulses propagate and ultimately enter synapses, which allow their ability to be increased or decreased. The most interesting result though is that there is plasticity in a neural network [3].

The neurons display long-term shifts in the frequency of their partnerships in response to the stimulus pattern. New associations with other neurons can also be created by the neurons. Often even whole neuron collections can move from place to place. This processes provide the framework for brain learning. Brain can be used as an incredibly dynamic and non-linear information retrieval mechanism and a parallel one. Information is collected and analyzed continuously over the entire network and not at unique positions in a neural network. In other words, all data and computation in neural networks are global and not local [4].

Due to the plasticity, neurons are reinforced to provide the 'correct answer' and those that contribute to the 'false response' are diminished. In this way, neural networks can learn from experience [5]. The basic and important feature of neural biological networks is learning. The flexibility and naturalness with which you can learn led to attempts in a machine to imitate a biological neural network [6].

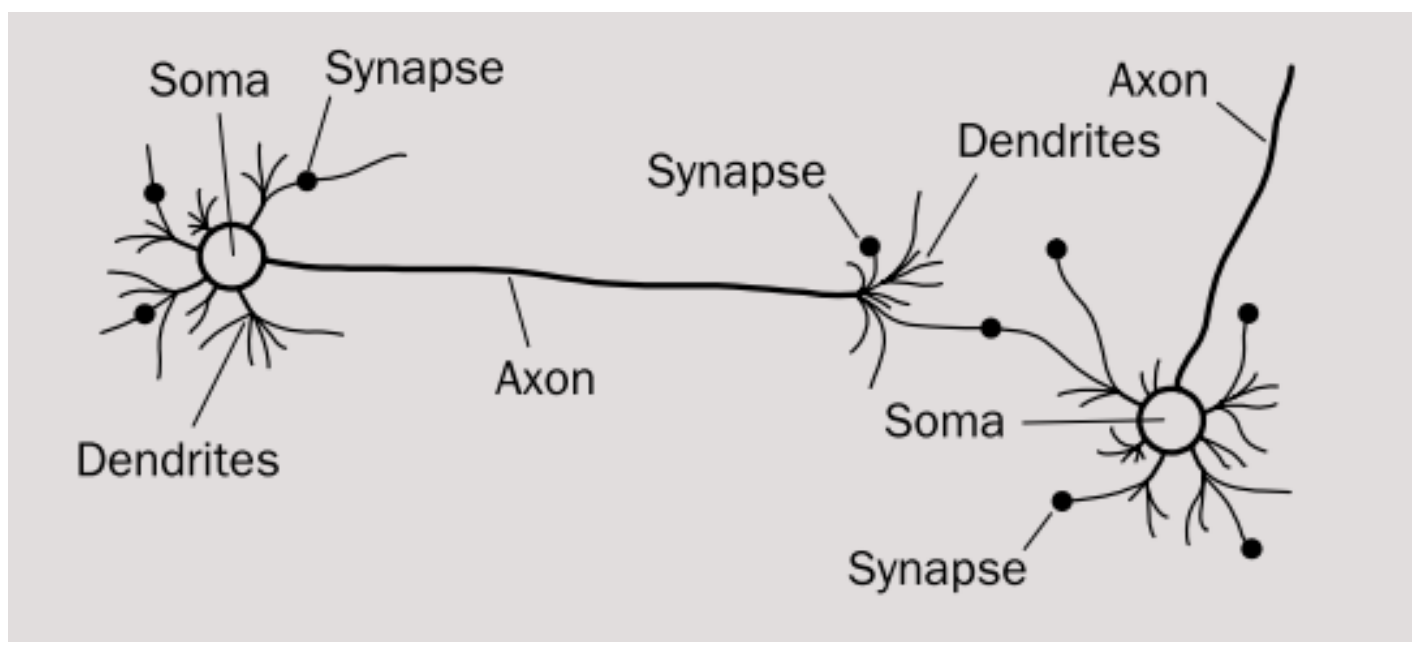

Figure 1: Biological neural network

Due to the plasticity, neurons are reinforced to provide the 'correct answer' and those that contribute to the 'false response' are diminished. In this way, neural networks can learn from experience [7]. The basic and important feature of neural biological networks is learning. The flexibility and naturalness with which you can learn led to attempts in a machine to imitate a biological neural network [8]. Due to the plasticity, neurons are reinforced to provide the 'correct answer' and those that contribute to the 'false response' are diminished. In this way, 
neural networks can learn from experience [9]. The basic and important feature of neural biological networks is learning. The flexibility and naturalness with which you can learn led to attempts in a machine to imitate a biological neural network.

An artificial neural network consists of many very basic and interconnected processors, also known as neurons, identical to the brain's biological neurons. The neurons are connected by a weighted relation between the neurons. Via its connections per neuron receives a variety of input signals but never generates more than one output signal. The output signal is conveyed by the output of the neuron (corresponding to the biological axon). The outgoing link divides into multiple branches that give the same signal (the signal is not divided among these branches in any way). The outgoing branches stop at the incoming neurons of the network. The relations of a standard ANN are seen in Figure 2.

The neurons are interconnected and each relation has a numerical weight. Weights are the fundamental means of long lasting ANN recall. They convey the power of each neuron input, or in other words its value. A neural network 'learns' through repetitive weight changes [10] [11].

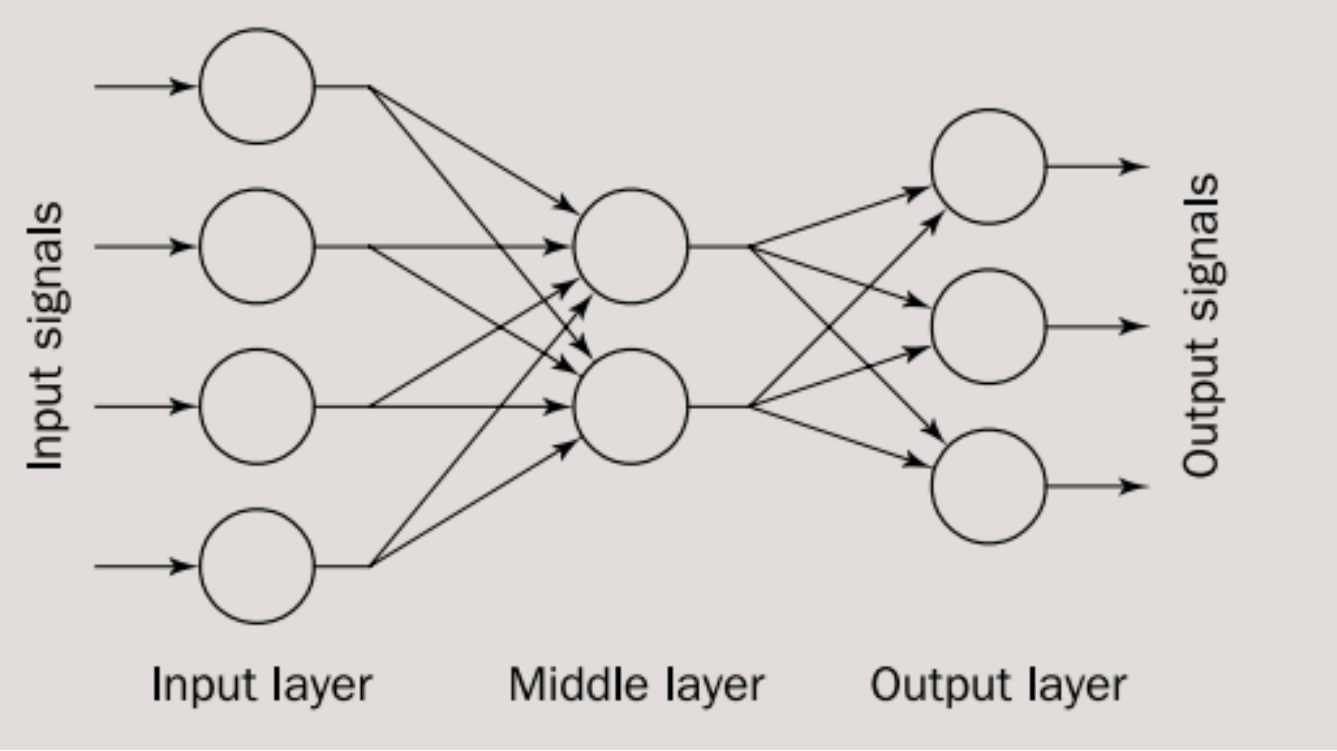

Figure 2: Architecture of an usual artificial neural network

\section{Weight Adjustment and outputs}

A standard ANN, as seen in figure 2, consists of a hierarchy of layers, and these layers include neurons in the networks. Input and output layers are connected to the external world. The size is changed such the weight is in alignment with the behavior of the input/output network. Every 
neuron is a simple processing unit for the information. In terms of inputs and numerical weight, it can measure its activation level. One must first determine how many neurons to use and how neurons should be connected to form a network in order to create the artificial neural network. This means that the network architecture must be chosen first. We then determine which algorithm to use to learn [4] [12].

Finally, the neural network are trained, which involves weighing down the network and updating weights from a variety of training cases. A neuron receives several signals in its input connections, determines a new degree of activation and sends it across output links as a signal. The signal input can be raw or other neuron outputs. Either a final solution to the problem or an input to other neurons will be the output signal [13] [14]. A normal neuron appears in Figure 3.

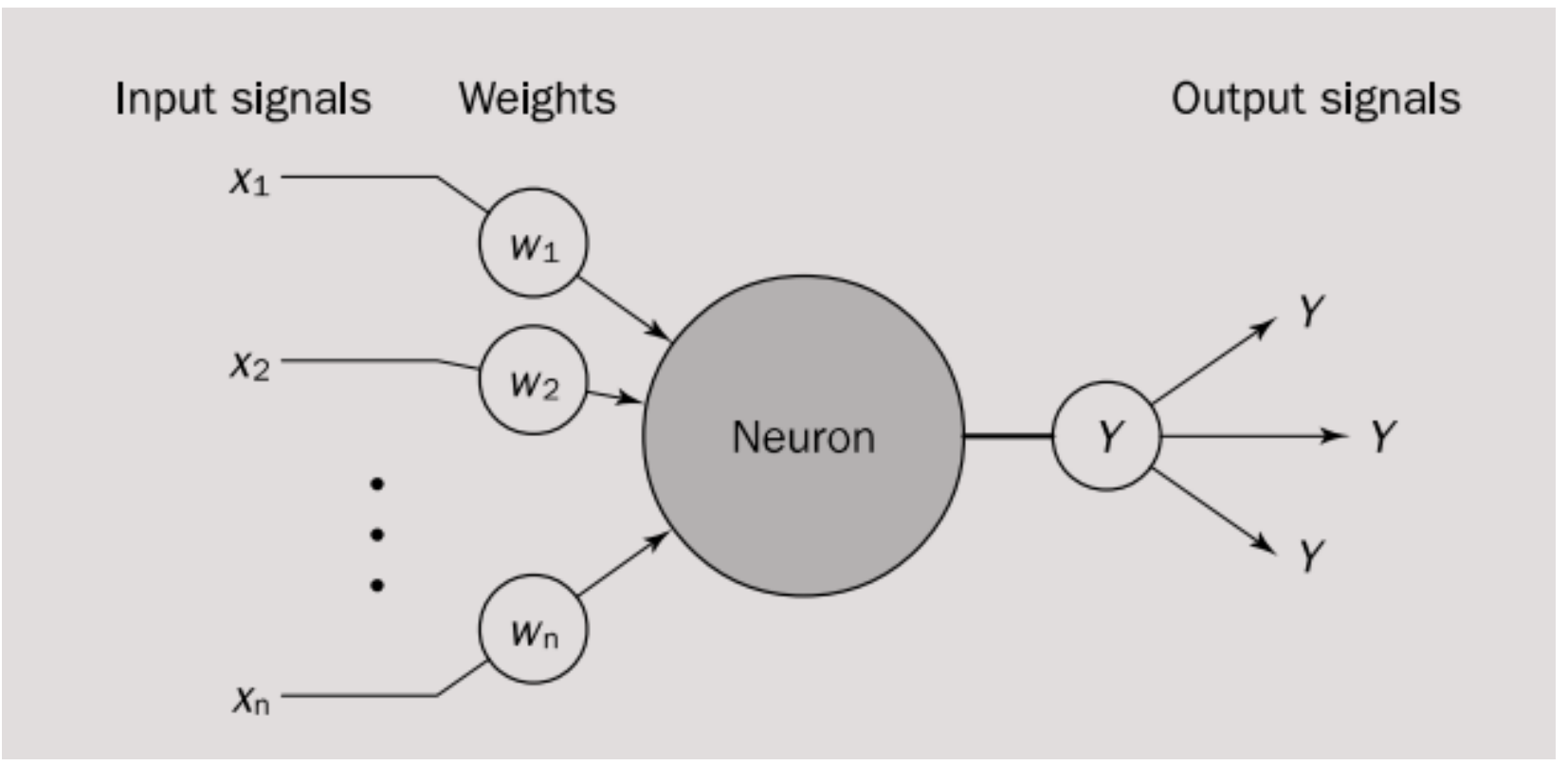

Figure 3: Diagram of a neuron

The neuron measures the weighted sum of the signal input and compares the result to a threshold value. If the net input is below the threshold, the corresponding neuron is 1 . The neuron is activated, and the output achieves a value of S 1 if the net input is greater than or equal to the threshold [15] [5]. The neuron uses the following function of transition or activation:

Equation 1: 


$$
\begin{aligned}
& X=\sum_{i=1}^{n} x_{i} w_{i} \\
& Y= \begin{cases}+1 & \text { if } X \geqslant \theta \\
-1 & \text { if } X<\theta\end{cases}
\end{aligned}
$$

If $X$ is the net weighted neuron input, $\mathrm{xi}$ is the input value I $\mathrm{i}^{\prime} \mathrm{s}$ the input weight $\mathrm{I} \mathrm{n}$ is the neuron input number, and $\mathrm{Y}$ is the neuron output. The activation function of this form is called a sign function. The actual neuron outcome can therefore be interpreted as the signal activation function

Equation 2:

$$
Y=\operatorname{sign}\left[\sum_{i=1}^{n} x_{i} w_{i}-\theta\right]
$$

\section{The novel Models}

\section{Modeling and prediction of dust source Hybridized neural fuzzy [16]}

Dust storms are known to play an important role in multiple climatic, geochemical and environmental cycles. The environment would have a direct effect on public health and harm the environment. The identification of dust sources areas therefore plays a key role in controlling the effects of this hazard. The work is the first attempt to categorize areas of dust sources using hybridized machine learning algorithms.

Each hybridized model is designed as an intelligent system consisting of the Adaptive NFIS, integrated with a combination of algorithms of metaheuristic optimization: bat algorithm (BA), cultural algorithm (CA), and differing evolutions (DE). The hybridized model integrates data from two main sources - the Moderate Resolution Imaging Spectroradiometer (MODIS) Deep Blue and the Ozone Monitoring Instrument (OMI), as well as specific data from field surveys and dust samples. 
Good fit analysis is performed to assess the hybridized model's predictive capacity using different statistical criteria, including true statistical skills (TSS) and the area under the recipient's characteristic curve (AUC). The findings show that the hybrid ANFIS-DE model (with AUC 1/4 84.1 percent, TSS 1/4.73) exceeds the other hybridized models for dust storm forecasting.

\section{Design and Application of Mixed Natural Gas Monitoring System [17]}

One of the key technologies in the exploitation and usage of natural gas is the analysis of natural gas components. For the gas extraction industry, a stable and precise online monitoring system for natural gas is needed. The model developed a new hardware architecture online monitoring system for natural gas. It increases the system's reliability and maintenance.

The software and hardware coordination is supported by a specific instruction set. The EWMA approach is used to pre-process the sensor array's raw real-time databy exponentially weighted moving average to reduce noise. A custom neural network for calibration is designed. And there is evidence of an optimum solution for accuracy and hardware scales that the relationship between performance and structure is the gas neural network.

The design focuses not only on optimizing individual components but also on improving the system. For several months the system has been operating stably in the gas fields. It meets stability, ease of use, maintenance and online surveillance requirements for industrial applications.

\section{Predicting the maturity and organic richness [18]}

For any source rock that is considered to be key parameters for evaluation of the potential of the organic carbon (TOC) and maturity level (Tmax). The TOC and Tmax are mainly estimated through analyzing core samples or cuttings using the common acidification and pyrolysis nonfilter combustion, both methods time consuming and expensive. In recent years, therefore, the literature focuses on the search for fast, cheap and adequate methods. The purpose of the model is to develop an accurate model for TOC and TMAx based on profounds, sonic natural gamma of deep resistivity of petrophysical depth logs and main-piece analysis (PCA). Apply artificial neural networks.

However, it is difficult to rely on petrophysical properties alone, involving difficult to use mathematical relations and dealing with huge data, which may not have a broad application, which means that the use of ANN has become a necessity. The data were collected for 16 puits 
representing a tight gas reservoir in Northeastern British Columbia Canada in Britain's British Columbia Oil Gas Commission (BCGC). The use of ANN in the model in combination with PCA has demonstrated its reliability and effectiveness in TOC and Tmax predictions.

For nine input parameters, results from the retro- propagation and BR regularisation technique $(\mathrm{R} 2=94 \%$ and $89 \%)$ in the training and validation phases produced satisfactory performance at TOC predictions and for the Tmax $(\mathrm{R} 2=88 \%$ and $86 \%)$ with 5 input parameters during the training and validation phases respectively ANN models were tested and validated on 2 wells which were randomly selected with the same log dataset, but did not participate in the main training and testing processes.

The test results were extremely high, for TOC and Tmax R2 $=88,71 \%$ and TOC $85,39 \%$ in well one respectively, and R2 $=85,19 \%$ and TOC and Tmax 84,28\% for well two respectively. The results of the model demonstrate that the use of ANN is practicable and can be used in applications that evaluate organic wealth and maturity of the target source rock using conventional logs, particularly when geochemical datasets are inadequate or not yet complete.

\section{COVID-19 genome analysis [19]}

In the first half of January 2020, approximately a month after the genome of the novel coronavirus (COVID-19) disease, Wuhan, the capital of the province of Hubei, China, was sequenced. The genome sequencing of COVID-19 is essential for understanding the virus conduct, the origin, the rate of its mutation, medication/vaccines development and effective prevention strategies.

The paper examines the use of artificial intelligence techniques to learn important information from genome sequences of COVID-19. Sequential pattern (SPM) mining is applied to a COVID-19 genome corpus first to determine whether or not it is possible to find interesting hidden patterns that reveal the frequent patterns and the relationships of nucleotide bases. Second, sequence prediction models are used to evaluate whether nuclear base(s) from earlier model(s) can be predicted.

Thirdly, an algorithm is designed to identify places in genome sequences in which base nucleotides are changed and calculated for the mutation rate for mutation analysis in genome sequences. The results show that SPM and methods of mutation analysis can disclose interesting information and patterns in the COVID-19 genome sequences for examining developments and variations respectively in COVID-19 strains. 


\section{Prey Predator Algorithm for Prediction of the COVID-19 [20]}

The worldwide spread of the COVID-19 epidemic has led to various investigations, including the estimation of anticipated cases. It helps to determine the need to deal with pandemic cases. The researchers used artificial neural networks (ANNs) in the model to predict the number of COVID-19 cases in Brazil and Mexico in the next few days. The algorithm for predator prey (PPA) is used for model trainings as a metaheuristic algorithm.

The proposed performance of the ANN models was analyzed by the Root Middle Squared Fehler (RMSE) (R). The best prediction of infections (active cases), recoveries and deaths in Brazil and Mexico is demonstrated by the ANN model. The ANN-models show very well predicted values in their simulation results. The ANN's prediction errors are significantly lower in percentages with metaheuristic algorithms than traditional monolithic neural networks. The model shows that Brazil and Mexico will receive daily infections, recoveries and deaths early in 2021 .

\section{Assessing and predicting air quality during the lockdown due to the COVID-19 [21]}

The model deals with the simulation, prediction and use, before and during the spread of the pandemic of the Virus COVID-19 by an artificial neural network, of air pollutant in the city of Irbid (ANN). Based on data obtained at the 2019 and the first quarter of the year 2020 air quality monitoring stations, a simulation and prediction of concentrations of three air pollutants - NO2, sulfur dioxide (SO2) and particulate matter with a diameter of less than $10 \mu \mathrm{m}$, could be developed using an ANN model(PM10).

Several ANN model configurations were tested to select the best model to estimate the concentration of the three air pollutants that are used as input to the model using meteorological parameters. The findings show that during coronavirus-locking, the concentration of pollutants was decreased by several percentages compared to their levels before the pandemic period (from $29 \%$ for PM10 to $72 \%$ for $\mathrm{NO} 2$ ).

Moreover, the ANN model, developed during the pandemic period, can simulate and estimate the pollutant concentration with sufficient precision as assessed by determination coefficient values and the mean square error. The results of the model show that adequately trained and structured ANN can be a useful instrument to accurately predict air quality parameters. 


\section{Prediction of the confirmed cases and deaths of global COVID-19 using artificial intelligence [22]}

The 2019 coronaviral outbreak (COVID-19) has affected the environment, the environment, the economy, society and human health significantly. The prediction and analysis of confirmed events and deaths in COVID-19 has become an important task, given the increasingly severe global epidemic dynamics. The model constructs a network of artificial neurons (ANN) for modeling confirmed COVID-19 cases. The World Health Organization is collecting confirmed cases and death data from 20 January until 11 November 2020. (WHO).

The statistical indicators of the prediction model are verified and assessed by introducing a root mean square error (RMSE), a correlation coefficient (R) and a mean absolute error (MAE). The size of the confirmed cases of training and testing and death base used in the model is optimized. The best simulatory performance in training and test dataset with RMSE, R and MAE is achieved with the 7 past days as input variables. The estimated $R$ is respectively 0.9948 and 0.9683. Experimental simulation shows better performance than other algorithms when reproducing the number of confirmed cases and deaths, compared to various algorithms.

The model shows that the ANN model is appropriate for future prediction of confirmed COVID-19 cases and deaths. The model also predicts confirmed COVID-19 cases and deaths from 5 June 2020 until 11 November 2020, using the ANN model. During the period predictable, R, RMSE and MAE are 0.9848, 17.554 and 12.229 in the case of new confirmed COVID-19 cases, R, RMSE and MAE respectively in 0.8593, 631.8 and 463.7 in the case of newly confirmed COVID-19 deaths. The confirmed cases and deaths of COVID-19 are very similar to those that are actually confirmed and deaths. The results show that strict controls to prevent the further spread of the epidemic should be implemented.

\section{Conclusion}

Data are one of the most important elements in the world today to advance society and to establish industrial technologies and sound public health. The Big Data climate has changed our everyday life significantly, as well as the economic and commercial landscape, with the help of Internet applications. Advanced techniques are necessary in order to handle and use these data profitably. A number of techniques which are particularly suited for these conditions are included in Artificial Intelligence (AI). Consequently, we discuss most recent ANN applications in view of the variety of methods and models, applications as well as data types. The most notable finding is that the AI application on public health is increasing. 


\section{References}

[1] M. O. Okwu and L. K. Tartibu, "Artificial Neural Network," in Studies in Computational Intelligence, 2021.

[2] L. Gatys, A. Ecker, and M. Bethge, “A Neural Algorithm of Artistic Style,” J. Vis., 2016.

[3] M. Majumder, "Artificial Neural Network," 2015.

[4] B. Yegnanarayana, "Artificial neural networks for pattern recognition," Sadhana, 1994.

[5] S. J. Kwon, Artificial neural networks. 2011.

[6] A. Intelligence, "Fund amentals of Neural Networks Artificial Intelligence Fundamentals of Neural Networks Artificial Intelligence," Fundam. Neural Networks AI Course Lect. 37 - 38, notes, slides, 2010.

[7] T. G. Clarkson, "Introduction to neural networks," Neural Netw. World, 1996.

[8] S. Albawi, T. A. Mohammed, and S. Al-Zawi, "Understanding of a convolutional neural network," in Proceedings of 2017 International Conference on Engineering and Technology, ICET 2017, 2018.

[9] M. I. Jord an and C. M. Bishop, "Neural networks," in Computer Science Handbook, Second Edition, 2004.

[10] Y. Goldberg, "A primer on neural network models for natural language processing," $J$. Artif. Intell. Res., 2016.

[11] P. M. Buscema, G. Massini, M. Breda, W. A. Lodwick, F. Newman, and M. AsadiZeydabadi, "Artificial neural networks," in Studies in Systems, Decision and Control, 2018.

[12] M. H. Hassoun, "Fundamentals of Artificial Neural Networks," Proc. IEEE, 2005.

[13] B. L. Yoon, “Artificial neural network technology,” ACM SIGSMALL/PC Notes, 1989.

[14] P. Tino, L. Benuskova, and A. Sperduti, "Artificial neural network models," in Springer Handbook of Computational Intelligence, 2015.

[15] M. E. Yalçın, T. Ayhan, and R. Yeniçeri, “Artificial neural network models,” in 
SpringerBriefs in Applied Sciences and Technology, 2020.

[16] O. Rahmati et al., "Hybridized neural fuzzy ensembles for dust source modeling and prediction," Atmos. Environ., vol. 224, p. 117320, 2020.

[17] J. Wang et al., "Design and Application of Mixed Natural Gas Monitoring System Using Artificial Neural Networks," Sensors, vol. 21, no. 2, p. 351, 2021.

[18] A. Barham, M. S. Ismail, M. Hermana, E. Padmanabhan, Y. Baashar, and O. Sabir, "Predicting the maturity and organic richness using artificial neural networks (ANNs): A case study of Montney Formation, NE British Columbia, Canada," Alexandria Eng. J., vol. 60, no. 3, pp. 3253-3264, 2021.

[19] M. S. Nawaz, P. Fournier-Viger, A. Shojaee, and H. Fujita, "Using artificial intelligence techniques for COVID-19 genome analysis,” Appl. Intell., pp. 1-18, 2021.

[20] N. N. Hamadneh, M. Tahir, and W. A. Khan, "Using Artificial Neural Network with Prey Predator Algorithm for Prediction of the COVID-19: The Case of Brazil and Mexico," Mathematics, vol. 9, no. 2, p. 180, 2021.

[21] N. Shatnawi and H. Abu-Qdais, "Assessing and predicting air quality in northern Jord an during the lockdown due to the COVID-19 virus pandemic using artificial neural network," Air Qual. Atmos. Heal., pp. 1-10, 2021.

[22] Q. Guo and Z. He, "Prediction of the confirmed cases and deaths of global COVID-19 using artificial intelligence," Environ. Sci. Pollut. Res., pp. 1-11, 2021. 